\title{
Conversion surgery for hepatocellular carcinoma in the new era of targeted and immune checkpoint inhibitor therapies
}

\author{
Lei Zhao ${ }^{1}$, Haitao Zhao $^{2}$ \\ ${ }^{1}$ Department of Hepatobiliary Surgery, Shandong Cancer Hospital Affiliated to Shandong First Medical University, Jinan, China; ${ }^{2}$ Department of \\ Liver Surgery, Peking Union Medical College Hospital, Chinese Academy of Medical Sciences and Peking Union Medical College, Beijing, China \\ Correspondence to: Lei Zhao. Shandong Cancer Hospital Affiliated to Shandong First Medical University, No. 440 Jiyan Road, Jinan 250117, China. \\ Email: drzhaolei@hotmail.com; Haitao Zhao. Chinese Academy of Medical Sciences and Peking Union Medical College, No. 1 Shuaifuyuan, \\ Wangfujing, Beijing 100730, China. Email: Zhaoht@pumch.cn.
}

Submitted Sep 02, 2020. Accepted for publication Sep 12, 2020.

doi: 10.21037/hbsn-20-693

View this article at: http://dx.doi.org/10.21037/hbsn-20-693

In the recent decade, systemic treatment of unresectable hepatocellular carcinoma ( $\mathrm{uHCC}$ ) with targeted and immune checkpoint inhibitors (ICIs) has achieved great progress. In the SHARP study conducted 12 years ago, sorafenib, the first systemic drug approved by the Food and Drug Administration (FDA) for advanced HCC, increased the median overall survival (mOS) from 7.9 to 10.7 months (1). This year, in the newly-released results from phase III IMbrave 150 study, the combination of anti-programmed death ligand 1 (PD-L1) inhibitor atezolizumab and anti-vascular endothelial growth factor (VEGF) drug bevacizumab has significantly increased the mOS and median progress free survival (mPFS) compared to sorafenib group (2). In another combination that attracted much attention, a phase Ib study for uHCC found that lenvatinib plus pembrolizumab achieved a mOS of 22.0 months (3). Based on promising phase $1 \mathrm{~b}$ result, the phase III LEAP-002 study (NCT03713593) of lenvatinib plus pembrolizumab versus lenvatinib plus placebo as firstline treatment of uHCC is ongoing. However, although significant progress has been made, long-term survival in uHCC patients achieved by targeted and/or ICIs therapies is still very rare and patients surviving over 5 years are only found in limited case reports.

Radical therapies (hepatic resection, liver transplantation and ablation in well-selected patients) are still the only curative-intent treatments for HCC. In patients who meet guidelines and undergo resection, 5-year survival rate with these modalities is over $60 \%$ (4). However, just only $10-30 \%$ of HCC patients are ideal candidates for liver resection, and $70 \%$ of them will experience recurrence within 5 years of resection (4).

Taking these data together, a very straightforward conclusion will be arrived at; if more uHCC patients can have curative therapy after conversion surgery, and less patients develop recurrence by adjuvant therapies, significantly more HCC patients will experience long-term survival or even cure. At new era, targeted drugs, ICIs or its combination therefore bring new hope for both conversion surgery and adjuvant therapy.

Conversion surgery in HCC is not a new concept. Tang et al. reported that from 1958 to 2003, 139 (12.8\%) out of 1085 uHCC patients were converted to "downstaging resection", and their 5-year survival rate reached $48.7 \%$ (5). The conversion therapies included hepatic artery ligation (HAL), hepatic artery cannulation with chemotherapeutic infusion (HAI), radiotherapy and radioimmunotherapy or various combinations therein. Fan et al. from the same institute reported a conversion rate of $18.1 \%$ (65 out of $360)$ in uHCC patients by transarterial chemoembolization (TACE), and a 5 -year survival rate was $56.0 \%$ (6). Excepted hepatectomy, downstaging resection of $\mathrm{uHCC}$ for liver transplantation is also promising strategy (7).

The objective response rate (ORR) is pivotal for the conversion surgery. ORR of TACE for intermediate-stage HCC is reported to be $52.5 \%$ (4); compare to TACE, the ORR of sorafenib in SHARP study was only $2 \%$ [7 partial responses (PR) out of 299 advanced HCCs]; later, the IMbrave 150 study, reported an ORR of sorafenib at $13.3 \%$ [3 complete responses (CR) and 18 PR out of 158 
Table 1 Reported conversion surgery of HCC following TKI/ICI in the literature

\begin{tabular}{|c|c|c|c|}
\hline & References & Case number & Combined therapies \\
\hline 1 & World J Gastroenterol 2011;17:2255 & 1 & Gemcitabine plus oxaliplatin \\
\hline 2 & Int J Hepatol 2011:791013 & 2 & \\
\hline 3 & Liver Int 2011;31:740 & 2 & \\
\hline 5 & Gastroenterol 2015;8:300 & 1 & $\mathrm{RT}$ \\
\hline 6 & Gan To Kagaku Ryoho 2015;42:1638 & 1 & TAE/TACE \\
\hline 7 & Anticancer Res 2018;38:501 & 2 & \\
\hline 8 & Exp Clin Transplant 2018;16:227 & $2 / 5$ & Convert to $\mathrm{LT}$ \\
\hline \multicolumn{4}{|c|}{ Lenvatinib } \\
\hline 1 & Onco Targets Ther 2019;12:7355 & 1 & PD-1 \\
\hline 2 & Anticancer Res 2019;39:5695 & 1 & \\
\hline 3 & Liver Cancer 2020;9:358 & 1 & \\
\hline \multicolumn{4}{|c|}{ Regorafenib } \\
\hline 1 & Clin J Gastroenterol 2020;13:428 & 1 & Following sorafenib \\
\hline
\end{tabular}

TACE, transarterial chemoembolization; RT, radiotherapy; LT, liver transplantation; TAI, transarterial infusion; PD-1, anti-programmed cell death protein 1.

uHCCs] according to the modified Response Evaluation Criteria in Solid Tumors (mRECIST). Four years after its approval by FDA, sporadic cases of conversion surgery following sorafenib began to be reported (Table 1). Over the last 13 years, there are only few reports of conversion surgery following sorafenib. The ORR of lenvatinib in the REFLECT study was $24.1 \%$ (6 CR and 109 PR out of $478 \mathrm{uHCCs}$ ) (8). Lenvatinib received FDA approval for the first line treatment of uHCC in 2018, so far there have been 3 conversion surgeries reported (Table 1). There was a case report that PD-1 inhibitor could as a downstaging therapy for liver transplantation for uHCC (9). The ORR of atezolizumab plus bevacizumab in IMbrave 150 study was $33.2 \%$ ( $33 \mathrm{CR}$ and $75 \mathrm{PR}$ out of $325 \mathrm{uHCCs}$ ) (2), and the ORR of lenvatinib plus pembrolizumab in phase $\mathrm{Ib}$ study reached as high as $46 \%$ (11 CR and 35 PR out of 100 uHCCs) (3). On $29^{\text {th }}$ May 2020, FDA has approved Atezolizumab plus bevacizumab for the first line treatment of uHCC.

Both the mOS and the ORR of these new tyrosine kinase inhibitor (TKI) + ICI treatments in HCC have reached the similar level as TACE, we should therefore be confident that more and more conversion surgeries can be expected with these therapies. In the 2020 ASCO (American Society of Clinical Oncology) Conference, Sun et al. reported that out of 60 consecutive uHCC patients, 11 patients (18.3\%) were converted to resectable through different TKI plus anti-PD-1 therapy, and 9 patients underwent hepatectomy. Notably, among 9 patients, there were 5 PR and no CR evaluated before surgery, there were 3 instances stable diseases (SD) with one patient experiencing progressive disease (PD) (RECIST v1.1), but the uHCC was still converted to resectable and hepatectomy was achieved successfully (10).

In clinical trials of target drugs and ICIs, OS is often the main focus and primary endpoint, because PFS, ORR and time to progression (TTP) are regarded as not consistently predictive of OS in HCC. This makes sense from the simple medical oncological viewpoint. While from the multidisciplinary viewpoint, longer term survival or even 
curative outcome can be achieved through the combination of different therapeutic modalities. In this setting, ORR of target drugs and ICIs in systemic treatment of uHCC can be potentially very important. Considering the fact that the TKI + ICI treatments in uHCC has led to the ORR and $\mathrm{mOS}$ close to TACE and other traditional therapies like HAL + HAI, the systemic application of targeted drugs and/or ICIs in the conversion surgery of uHCC should be seriously considered, and well-designed randomized control trials (RCTs) should be organized to address this issue We hope another 10 to 20 percent of uHCC could be converted to curative $\mathrm{HCC}$ resection in the new era of targeted and immune checkpoint inhibitor therapies. In the future, we anticipate the results of immunotherapy 3.0 era (PD-1/PD-L1+TKI/VEGF + locoregional therapy) for uHCC (NCT03778957/EMERALD-1; NCT04246177/ LEAP-012; NCT04224636/DEMAND). Identification and enrichment of target patients (PD-L1 positive, good ECOG score or local advanced stage) need for further investigation.

\section{Acknowledgments}

We thank Dr. Alex Gordon-Weeks from Nuffield Department of Surgical Sciences, University of Oxford for his careful reviewing and constructive suggestions to the manuscript.

Funding: LZ is supported by the National Natural Fund of China (No. 30801383 and No. 81472713).

\section{Footnote}

Provenance and Peer Review: This article was commissioned by the editorial office, Hepatobiliary Surgery and Nutrition. The article did not undergo external peer review.

Conflicts of Interest: Both authors have completed the ICMJE uniform disclosure form (available at http://dx.doi. org/10.21037/hbsn-20-693). HZ serves as an unpaid editorial board member of Hepatobiliary Surgery and Nutrition. The other author has no conflicts of interest to declare.

Ethical Statement: The authors are accountable for all aspects of the work in ensuring that questions related to the accuracy or integrity of any part of the work are appropriately investigated and resolved.

Open Access Statement: This is an Open Access article distributed in accordance with the Creative Commons
Attribution-NonCommercial-NoDerivs 4.0 International License (CC BY-NC-ND 4.0), which permits the noncommercial replication and distribution of the article with the strict proviso that no changes or edits are made and the original work is properly cited (including links to both the formal publication through the relevant DOI and the license). See: https://creativecommons.org/licenses/by-nc-nd/4.0/.

\section{References}

1. Llovet JM, Ricci S, Mazzaferro V, et al. Sorafenib in advanced hepatocellular carcinoma. N Engl J Med 2008;359:378-90.

2. Finn RS, Qin S, Ikeda M, et al. Atezolizumab plus Bevacizumab in Unresectable Hepatocellular Carcinoma. N Engl J Med 2020;382:1894-905.

3. Finn RS, Ikeda M, Zhu AX, et al. Phase Ib Study of Lenvatinib Plus Pembrolizumab in Patients With Unresectable Hepatocellular Carcinoma. J Clin Oncol 2020;38:2960-70.

4. Villanueva A. Hepatocellular Carcinoma. N Engl J Med 2019;380:1450-62.

5. Tang ZY, Zhou XD, Ma ZC, et al. Downstaging followed by resection plays a role in improving prognosis of unresectable hepatocellular carcinoma. Hepatobiliary Pancreat Dis Int 2004;3:495-8.

6. Fan J, Tang ZY, Yu YQ, et al. Improved survival with resection after transcatheter arterial chemoembolization (TACE) for unresectable hepatocellular carcinoma. Dig Surg 1998;15:674-8.

7. Yao FY, Fidelman N. Reassessing the boundaries of liver transplantation for hepatocellular carcinoma: Where do we stand with tumor down-staging? Hepatology 2016;63:1014-25.

8. Kudo M, Finn RS, Qin S, et al. Lenvatinib versus sorafenib in first-line treatment of patients with unresectable hepatocellular carcinoma: a randomised phase 3 noninferiority trial. Lancet 2018;391:1163-73.

9. Schwacha-Eipper B, Minciuna I, Banz V, et al. Immunotherapy as a downstaging therapy for liver transplantation. Hepatology 2020. [Epub ahead of print].

10. Sun HC, Zhu XD, Huang C, et al. Initially unresectable hepatocellular carcinoma treated by combination therapy of tyrosine kinase inhibitor and anti-PD-1 antibody followed by resection. J Clin Oncol 2020;38:e16690.

Cite this article as: Zhao L, Zhao $\mathrm{H}$. Conversion surgery for hepatocellular carcinoma in the new era of targeted and immune checkpoint inhibitor therapies. HepatoBiliary Surg Nutr 2020;9(6):809-811. doi: 10.21037/hbsn-20-693 